\title{
Genome-wide analysis and characterization of F-box gene family in Gossypium hirsutum
}

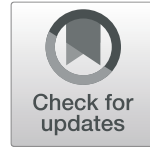

$\mathrm{L}$

Shulin Zhang ${ }^{1,2}$, Zailong Tian' ${ }^{1}$, Haipeng Li ${ }^{1}$, Yutao Guo ${ }^{1}$, Yanqi Zhang ${ }^{1}$, Jeremy A. Roberts ${ }^{3}$, Xuebin Zhang ${ }^{1 *}$ and Yuchen Miao ${ }^{1 *}$

\begin{abstract}
Background: F-box proteins are substrate-recognition components of the Skp1-Rbx1-Cul1-F-box protein (SCF) ubiquitin ligases. By selectively targeting the key regulatory proteins or enzymes for ubiquitination and 265 proteasome mediated degradation, F-box proteins play diverse roles in plant growth/development and in the responses of plants to both environmental and endogenous signals. Studies of F-box proteins from the model plant Arabidopsis and from many additional plant species have demonstrated that they belong to a super gene family, and function across almost all aspects of the plant life cycle. However, systematic exploration of F-box family genes in the important fiber crop cotton (Gossypium hirsutum) has not been previously performed. The genomewide analysis of the cotton F-box gene family is now possible thanks to the completion of several cotton genome sequencing projects.
\end{abstract}

Results: In current study, we first conducted a genome-wide investigation of cotton F-box family genes by reference to the published F-box protein sequences from other plant species. $592 \mathrm{~F}$-box protein encoding genes were identified in the Gossypium hirsutume acc.TM-1 genome and, subsequently, we were able to present their gene structures, chromosomal locations, syntenic relationships with their parent species. In addition, duplication modes analysis showed that cotton F-box genes were distributed to 26 chromosomes, with the maximum number of genes being detected on chromosome 5. Although the WGD (whole-genome duplication) mode seems play a dominant role during cotton F-box gene expansion process, other duplication modes including TD (tandem duplication), PD (proximal duplication), and TRD (transposed duplication) also contribute significantly to the evolutionary expansion of cotton F-box genes. Collectively, these bioinformatic analysis suggest possible evolutionary forces underlying F-box gene diversification. Additionally, we also conducted analyses of gene ontology, and expression profiles in silico, allowing identification of F-box gene members potentially involved in hormone signal transduction.

Conclusion: The results of this study provide first insights into the Gossypium hirsutum F-box gene family, which lays the foundation for future studies of functionality, particularly those involving F-box protein family members that play a role in hormone signal transduction.

Keywords: Gossypium hirsutum L., Cotton, F-box gene family, Ubiquitination, Protein degradation

\footnotetext{
* Correspondence: xuebinzhang@henu.edu.cn; miaoych@henu.edu.cn

${ }^{1}$ State Key Laboratory of Cotton Biology, Institute of Plant Stress Biology,

School of Life Sciences, Henan University, Jinming Street, Kaifeng 475004,

China

Full list of author information is available at the end of the article
}

(c) The Author(s). 2019 Open Access This article is distributed under the terms of the Creative Commons Attribution 4.0 International License (http://creativecommons.org/licenses/by/4.0/), which permits unrestricted use, distribution, and reproduction in any medium, provided you give appropriate credit to the original author(s) and the source, provide a link to the Creative Commons license, and indicate if changes were made. The Creative Commons Public Domain Dedication waiver (http://creativecommons.org/publicdomain/zero/1.0/) applies to the data made available in this article, unless otherwise stated. 


\section{Background}

The Ubiquitin (Ub)/26S proteasome pathway is an important post-translational regulatory process in eukaryotes that marks unwanted or misfolded proteins for degradation. This pathway also serves to adjust the activities of key regulatory proteins, and such processes being used by cells to respond rapidly to intracellular signals and environmental stimuli $[1,2]$. Ubiquitination of target proteins occurs in the Ub/26S proteasome pathway predominantly via three enzymatic reactions. First, an ATP-dependent activation of ubiquitin is catalyzed by enzyme E1, then the activated ubiquitin is transferred to the ubiquitinconjugating enzyme E2, and, finally, the ubiquitin is selectively bound to substrate proteins directed by the ubiquitin-protein ligase E3. The E3 ligase in the Ub/26S proteasome pathway is essential for recognition of target proteins for ubiquitination, and is the specificity determinant of the E3 complex for appropriate targets [3]. To date, several hundred E3 ubiquitin ligases have been identified, one of the best characterized being the SCF protein complex consisting of RBX1, SKP1, CULLIN, and F-box proteins $[4,5]$. In this complex, RBX1, CULLIN1, and SKP1 are invariant, and interact together to form a core scaffold. SKP1 further interacts with a specific F-box protein. F-box proteins found within the SCF complexes vary significantly in sequence. As the name suggests, proteins in this family contain at least one conserved F-box motif of 4050 amino acids at their $\mathrm{N}$-terminus which interacts with the SKP1 protein. In contrast, the C-terminal region of Fbox proteins usually contain highly variable proteinprotein interaction domains which serve to specifically recruit substrate proteins for ubiquitination and subsequent 26S proteasome degradation. Therefore, F-box proteins play a crucial role for defining the specific substrates of the SCF complexes for destruction [6,7].

As a result of rapid advances in DNA sequencing technologies, hundreds of F-box genes have been identified in the genome of every plant species sequenced, including Arabidopsis [8], rice [8], poplar [8], soybean [9], Medicago [10], maize [11], chickpea [12], apple [13] and pear [14], respectively containing 692, 779, 337, 509, $359,285,517$, and 226 F-box genes. In addition to the $\mathrm{N}$-terminus F-box domain, the variable protein-protein interaction motifs found at the $\mathrm{C}$ termini of F-box proteins can be used to classify F-box proteins into different subfamilies based on the presence of interaction motifs such as leucine-rich repeats (LRR), Kelch, WD-40, Armadillo (Arm), tetratricopeptide repeats (TPRs), Tub, actin, DEAD-like helicase, and jumonji (JmjC) [15]. The large number of F-box proteins theoretically forms a diverse array of SCF complexes which, in turn, will recognize a wide range of substrate proteins for ubiquitination and degradation. Functional characterization of a limited number of plant F-box genes have demonstrated that F-box proteins are associated with many important cellular processes such as embryogenesis [16, 17], seed germination [18], plant growth and development [19, 20], floral development $[14,21]$, responses to biotic and abiotic stress [22-24], plant secondary metabolism [25-27], hormonal responses, and senescence [4, 28, 29].

Worldwide, cotton is an extremely important fiber crop. Upland cotton (Gossypium hirsutum) is the primary cultivated species, contributing more than $90 \%$ of global cotton fiber production [30-32]. Gossypium hirsutum is also one of the descendant allotetraploid species and is believed to be derived from polyploidization between a spinnable-fibercapable A genome species (Gossypium arboreum) and a non-spinnable-fiber-capable D genome species (Gossypium raimondii) [33]. Systematic exploration of F-box family genes in cotton (Gossypium hirsutum) had not been previously performed due to the incomplete state of cotton genome sequencing projects. Collectively, only a few F-box proteins have been functionally explored in Gossypium hirsutum, including two putative homologues of the MAX2 genes that have been shown to control shoot lateral branching in Arabidopsis [34]. In a second study, Wei et al. [35] cloned a GhFBO (GenBank:JF498592) gene containing two Tubby $\mathrm{C}$-terminal domains, and showed that this gene had elevated levels of expression in flower, stem, and leaf tissues. But the detailed biological function of GhFBO was not examined in their studies. With the completion of genome sequencing projects for an increasing number of cotton species, F-box protein encoding genes in Gossypium hirsutum have become amenable to a systematic investigation of their structures and syntenic relationships for further functionality studies.

In our current study, we present the results of a genome-wide analysis of F-box genes in Gossypium hirsutum. 592 F-box protein encoding genes were identified in the Gossypium hirsutume acc.TM-1 genome, and their gene structures, chromosomal locations, syntenic relationships across other cotton species, and duplication modes are presented, along with a discussion of the possible evolutionary effects on allotetraploid cotton F-box genes. Finally, we investigated gene ontology, the expression profiles of all F-box based on publicly available databases and the possible F-box gene members involved in hormone signal transduction. Our results provide the first overview of the Gossypium hirsutum F-box gene family, which we believe will lay the foundation for future functionality studies, particularly the F-box proteins that likely play important roles in hormone signal transduction.

\section{Methods}

\section{Identification and classification of F-box genes from Gossypium hirsutum}

To identify the F-box proteins from Gossypium hirsutum, the local BLASTP algorithm (with an E-value cut 
off of 1e-10) was applied to the Gossypium hirsutum genome database (http://mascotton.njau.edu.cn) [36] in a global search for F-box proteins. The initial query sequences were the 1808 previously published F-box protein sequences from Arabidopsis, Populus trichocarpa, and rice [8]. After this initial screening, all F-box protein candidates were verified by the Pfam (http://pfam.sanger.ac.uk/search) and SMART (http://smart.embl-heidelberg.de) webserver, with an e-value cut-off of less than 1.0 to ensure each candidate sequence contained at least one of the F-box motifs (PF00646, PF12937, PF13013, PF04300, PF07734, PF07735, PF08268 and PF08387). All proteins containing these F-box domains were considered to be F-box proteins from Gossypium hirsutum. According to their C-terminal proteinprotein interaction domains, the identified cotton F-box proteins were further classified into different subfamilies. In order to understand the evolution of the expansion of the cotton F-box genes, the F-box protein encoding genes from Gossypium raimondii and Gossypium arboreum were also identified and classified using the same approach.

\section{Dissection of different duplication modes of F-box genes from Gossypium hirsutum}

The MCScanX-transposed software package [37] was used to predict the genomic duplication mode of Gossypium hirsutum F-box genes, based on syntenic analyses comparing allotetraploid and corresponding diploids. Fbox genes within the Gossypium hirsutum genome were classified as transposed, proximal, tandem, or wholegenome duplications (WGD). First, the local BLASTP algorithm was used to compare Gossypium hirsutum versus Gossypium hirsutum, Gossypium hirsutum versus Gossypium raimondii, and Gossypium hirsutum versus Gossypium arboretum, for all F-box proteins from the $\mathrm{AD}, \mathrm{A} 2$ and $\mathrm{D} 5$ genome $(\mathrm{E}<1 \mathrm{e}-5$, top five matches and $\mathrm{m} 8$ format) without the scaffold gene. Second, the core program of MCScanX-transpose was executed using the BLASTP output (Gossypium hirsutum versus Gossypium raimondii, and Gossypium hirsutum versus Gossypium arboreum as the outgroup) and the annotation file (.ggf file) as the input. Finally, syntenic colinear gene pairs between allotetraploid and diploids, and the F-box gene from Gossypium hirsutum duplication mode were produced.

\section{Calculation of nonsynonymous (Ka) and synonymous (Ks)} substitution rates and $\mathrm{Ka} / \mathrm{Ks}$ ratios

Verified duplicated gene pairs originating from different duplication modes were used to calculate the $K a$ and $K s$ substitution rates. First, the coding sequences of duplicated genes were compared by LASTZ -master tools (http://www.bx.psu.edu/ rsharris/lastz) and an AXT file was produced. Then KaKs_Calculator 2.0 was used to estimate $K a$ and $K s$ values, and the $K a / K s$ ratios were calculated based on the AXT file with model-averaged method. The parameters were configured as described in the software package manuals $[38,39]$. The $\mathrm{Ka} / \mathrm{Ks}$ ratio was assessed to determine the molecular evolutionary rates of each gene pair. In general, $\mathrm{Ka} / \mathrm{Ks}<1$ indicates purifying selection; $\mathrm{Ka} / \mathrm{Ks}=1$ indicates neutral selection; and $\mathrm{Ka} / \mathrm{Ks}>1$ indicates positive selection. The divergence time of these gene pairs was estimated using the formula " $\mathrm{t}=\mathrm{Ks} / 2 \mathrm{r}$ ", with $\mathrm{r}\left(2.6 \times 10^{-9}\right)$ representing neutral substitution $[36,40]$.

\section{Gene ontology (GO) items and expression pattern analysis}

The GO annotation for cotton F-box protein encoding genes was obtained from the Gossypium hirsutum L. acc. TM-1 genome project [36]. The three top GO categories: molecular function (MF), biological process (BP), and cellular component $(\mathrm{CP})$ were analyzed. The functional annotations of F-box genes involved in any biological process (BP) were predicted based on putative homologues from Arabidopsis. thaliana. Expression data for all F-box protein-encoding genes were obtained from CottonFGD (https://cottonfgd.org/analyze) for 9 tissues (Calycle, Leaf, Petal, Pistil, Root, Stamen, Stem, Torus, fiber). The $\log 2$ transformed RPKM (reads per kilobase per million) values or TPM (transcripts copies per million tags) values were used to measure expression levels of the F-box genes, and to generate heat maps. Expression clusters were defined using Mev4.6.2 software (http://www.tm4.org/mev.html).

For in silico expression analyses, RNA-seq data for 8 Gossypium hirsutum L. acc. TM-1 tissues (torus, stem, leaf, root, 5dap fiber, 10dap fiber,15dap fiber and 25dap fiber) were downloaded from the NCBI SRA database (SRA available accession numbers SRX797899, SRX797900, SRX79901, SRX797902, SRX797917, SRX797918, SRX797919 and SRX797920 respectively [36]). All analyses were carried out using the Tophat-Cufflinks pipeline, with the following versions: Bowtie2 v2.3.4.3, Tophat v2. 1.1, Samtools v1.9 and Cufflinks v2.2.1. The G. hirsutum acc.TM-1 genome and gene model annotation file (GFF, gene. Ghir.NAU.gff3) downloaded from cotton gene (https://www.cottongen.org/) were used as reference. The FPKM values for F-box genes were utilized for K-means clustering using the XLSTAT version 2013 and standardized for generating the heatmaps using R software.

\section{Identification of F-box gene as the SCF complexes involved in hormone signal transduction pathway}

To identify the Gossypium hirsutum F-box genes which can potentially form the SCF complexes involved in plant hormone signal transduction pathways, we first obtained the protein sequences of the Arabidopsis F-box proteins involved in hormone signal transduction based on previous studies, including TIR1 in the auxin signaling pathway, $S L Y 1$ in the gibberellin signaling pathway, 
$E B F 2$ in the ethylene signaling pathway and the F-box genes that have been proposed to play a role in the ABA signaling pathway $[41,42]$. Second, we performed a local BLASTP algorithm-based search $(\mathrm{E}<1 \mathrm{e}-10$ and Identities $>50 \%$ ) against all F-box protein sequences using the above listed protein sequences from Arabidopsis as queries. From these results, a number of candidate Fbox genes likely involved in cotton IAA, JA, GA, ABA and ethylene signal transduction pathways were chosen, and their expression responses to different hormone treatments determined by qRT-PCR.

\section{RNA extraction and qRT-PCR}

To examine expression profiles of F-box protein encoding genes in hormone signal transduction pathways, Gossypium hirsutum L. acc. TM-1 leaves at the four-leaf stage were submerged in $100 \mu \mathrm{M} \mathrm{ABA}$ (Biotopped, cat number: A1049) solution, $100 \mu \mathrm{M}$ ACC (Ruitaibio) solution, and $100 \mu \mathrm{M}$ GA3 (Biotopped) solution, or were sprayed with $100 \mu \mathrm{M}$ IBA solution (Solarbio, cat number: 531A0214), respectively. Samples were collected from leaves at $0,1,3,6$, and $12 \mathrm{~h}$ after treatment. Samples collected at $0 \mathrm{~h}$ were used as controls. All samples were immediately frozen in liquid nitrogen and kept at $-80^{\circ} \mathrm{C}$ proir to total RNA extraction. Total RNA was extracted from the samples using the RNAprep Pure Kit (For Plants) (TIANGEN, Beijing, China). First-strand cDNA was synthesized based on reverse transcription of $1 \mu \mathrm{g}$ RNA digested by DNase I using the PrimeScript ${ }^{\mathrm{m}}$ RT Reagent Kit (Takara, Dalian, China). PCR amplifications were performed using $\mathrm{SYBR}^{\circ}$ Premix Ex Taq ${ }^{\mathrm{Tx}}$ (Takara). For real-time PCR, gene-specific primers were designed using Primer 5.0 (Additional file 5: Table S8). For the qRT-PCR assay, cDNA was diluted to $100 \mathrm{ng} / \mu \mathrm{L}$ with $\mathrm{ddH}_{2} \mathrm{O}$. The reaction (in a total volume of $20 \mu \mathrm{L}$ ) contains $10 \mu \mathrm{L}$ SYBR $^{\circ}$ Premix Ex $\operatorname{Taq}^{\text {ma }}(2 \times), 0.4 \mu \mathrm{L}$ of each primer $(10 \mu \mathrm{M}), 0.4 \mu \mathrm{l}$ ROX Reference Dye $(50 \times)$, $1 \mu \mathrm{L}$ template (about $100 \mathrm{ng} / \mu \mathrm{L}$ ), and $\mathrm{ddH}_{2} \mathrm{O}$ to make up the total volume. The qRT-PCR reaction was performed on a ROCHE Real-time PCR System (Applied Biosystems) as described [43]. Fold-changes were calculated using the comparative CT method (2- $\Delta \Delta \mathrm{Ct})$, using cotton GhActin1 as an internal reference [44].

\section{Results}

\section{Identification and classification of F-box genes in} Gossypium hirsutum

A total of 30,687 F-box encoding sequences were initially identified by local BLASTP. After the repetitive sequences were removed, 2904 sequences were retained, and were submitted to the Pfam and SMART webserver to confirm that the identified F-box proteins contained at least one of the established F-box domains. After this step, 592 cDNAs were ultimately verified as Gossypium hirsutum F-box genes, and were named based on their chromosomal locations. Gene names, IDs, chromosomal locations, exon numbers, amino acid composition, molecular weights and pIs are listed in Additional file 5: Table S1. In addition, 300 F-box genes from Gossypium raimondii and $282 \mathrm{~F}$-box genes from Gossypium arboreum were also separately identified using the same approaches (Additional file 5: Table S2 and Table S3). According to cotton origin and evolution studies [30-32, 45], the domesticated Gossypium hirsutum (allotetraploid AD-hybrid) species are the offspring formed between diploid cotton species Gossypium raimondii (D-genome) and Gossypium arboreum (A-genome). The polyploidization between the A-genome and $\mathrm{D}$-genome species leads to the tetraploid AD species containing two copies of the entire $\mathrm{A}$ and $\mathrm{D}$ genomes, which instead of two copies of each genome (one from each parent), has four (two from each parent). Interestingly, the $\mathrm{AD}$ offspring are quite different from both the parents in terms of fiber qualities, and stress and disease resistance, indicating that the $\mathrm{AD}$ genome rearrangements/ combinations have caused not only the genome size doubling but also potential gene expression changes. In our current studies, we found that Gossypium hirsutum possesses almost twice the number of F-box genes as compared to its diploid parents Gossypium arboretum and Gossypium raimondii, which indicates that most of the F-box genes are retained after polyploidization between the two diploid cotton species, Gossypium raimondii and Gossypium arboreum.

According to the functional domains found within the C-terminal region of the identified cotton F-box proteins, they can be grouped into 17 different subfamilies (Fig. 1). The F-box protein subfamily containing no-known C-terminal functional domains, designated as Fbox, is the largest cotton F-box gene subfamily containing 320 members. The remaining F-box proteins were divided into 16 subfamilies according to the presence of well-defined C-terminal functional domains, such as Actin ( 2 genes), ARM (7 genes), DUF (18 genes), FBA (46 genes), FBD/LRR (34 genes), FST_C (2 genes), JmJC (4 genes), Kelch (61 genes), LRR-Repeat (39 genes), Lysm (2 genes), PP2/PPR (12 genes), SCOP (3 genes), SEL1(4 genes), Tub (32 genes), WD40 (2 genes), and zf-MYNT (4 genes) (Fig. 1). It is interesting that, based on the Pfam database, the SCOP subfamily is present only in Gossypium hirsutum, and that the Herpes subfamily is absent in Gossypium hirsutum when compared with the F-box protein subfamilies in Gossypium raimondii and Gossypium arboreum. Three genes in the Gossypium hirsutum SCOP subfamily contain the cullin domain (PF00888) which usually are not present in plant F-box proteins. Cullin proteins, which are conserved in all eukaryotes, normally play roles as 


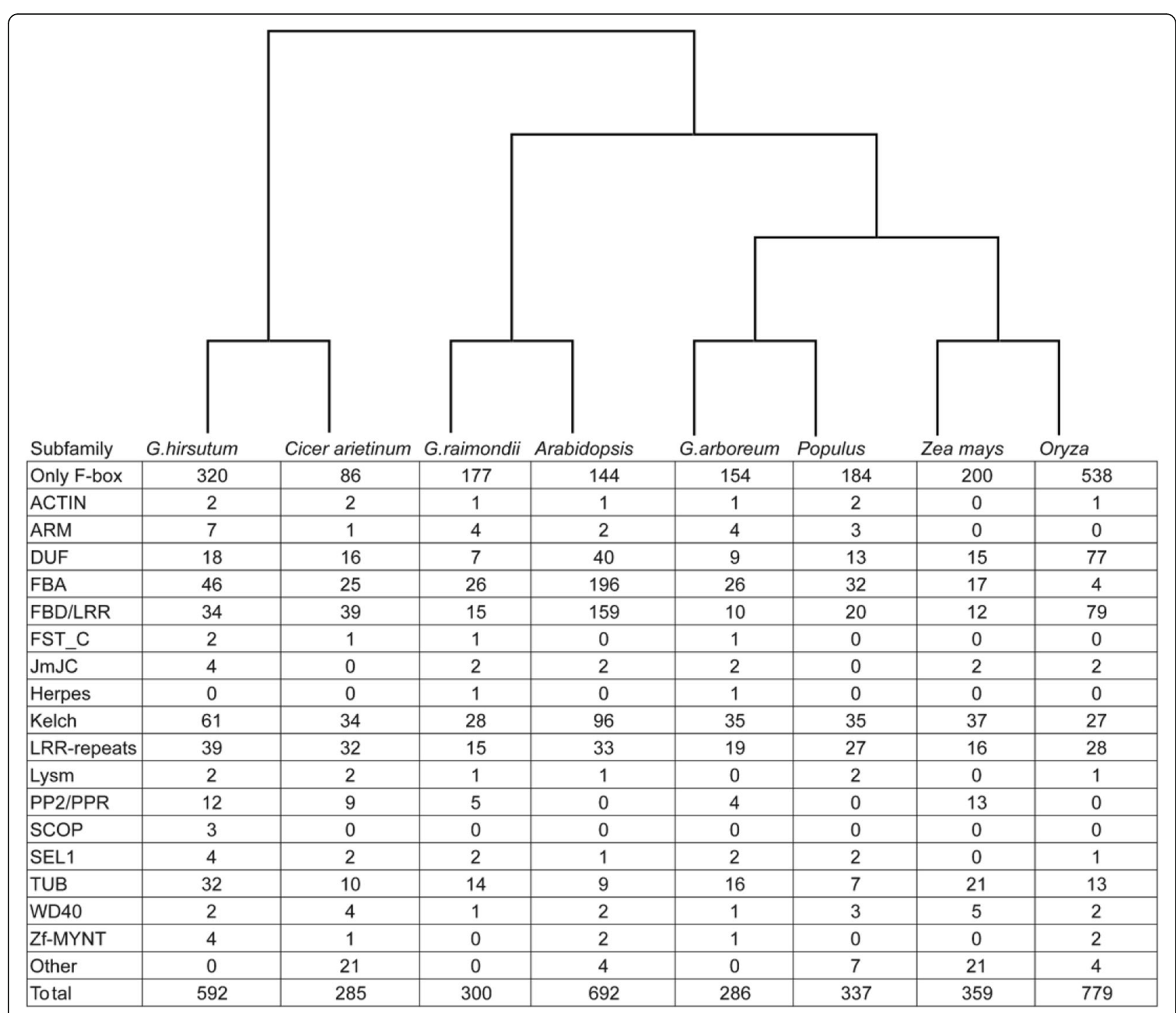

Fig. 1 The number and classicization of F-box genes identified in G. hirsutum, G.Raimondi and G.arboreum genomes. All the F-box genes were classified into different subfamilies based on their C-terminus functional domains (Pfam domains)

scaffold proteins supporting other components of the E3 ubiquitin ligase complexes. In the SCF complex, Cullin proteins usually link F-box proteins with the remaining members of SCF complexes, which likely allows the cotton SCOP F-box subfamily proteins to recruit their substrate proteins independently from the SCF complexes. In addition, the Herpes subfamily (Herpes_UL92(PF03048)) was only found in Gossypium raimondii and Gossypium arboreum, and not in Gossypium hirsutum, suggesting that Gossypium hirsutum experienced different forces of selection during cotton polyploidization [46]. Chromosomal breakages and rearrangements leading to different patterns of gene loss and gene retention during the polyploidization represents a possible explanation for this phenomenon [47].
The genomic distribution and gene expansion events of Gossypium hirsutum F-box genes

Using the genome sequence of Gossypium hirsutum acc.TM-1 as a reference, the $592 \mathrm{~F}$-box protein encoding genes were mapped to individual chromosomes or scaffolds. Of these, 524 F-box genes were assigned to 26 chromosomes, with the maximum number of genes being detected on chromosome 5 (37 genes), followed by chromosome 11 (36 genes), chromosome 18 (34 genes) and chromosome 21 (34 genes) respectively. Chromosome 4 contained the fewest F-box genes ( 6 genes), with the remaining $68 \mathrm{~F}$-box genes being located on unmapped scaffolds. Notably, longer chromosomes do not necessarily contain more F-box gene family members, indicating that the number of F-box genes on each 
chromosome is not correlated to length (Pearson correlation $\mathrm{r}=0.083 p$-value $=0.725$ ) (Fig. 2). This result demonstrates that cotton F-box protein encoding genes, like the F-box genes in other plant species, are unevenly distributed on the 26 chromosomes of Gossypium hirsutum [11, 12, 14, 15, 48].

When the genome from Gossypium arboreum (Agenome) and the genome from Gossypium raimondii (D-genome) were combined to produce the allotetraploid cotton AD genome, most of the cotton genes appear to have been duplicated at the whole genome level. To elucidate the evolutionary genome rearrangement and duplication patterns of the F-box protein encoding genes in Gossypium hirsutum, we performed a gene duplication event analysis including whole genome duplication (WGD), tandem duplication (TD), proximal duplication (PD) and transposed duplication (TRD) (Fig. 3). A total of 303 WGD F-box genes, corresponding to 166 duplicated gene pairs, were identified in Gossypium hirsutum which represents the largest portion of F-box genes in allotetraploid cotton, the number of WGD duplicated genes on each of the 26 Gossypium hirsutum chromosomes ranging from 0 on chromosomes 4 and 17 to 22 on chromosome 5 (Additional file 1 : Figure S1). 68 TD genes corresponding to 56 duplicated gene pairs, $30 \mathrm{PD}$ genes corresponding to 28 duplicated gene pairs and 53 TRD, including DNA transposed duplicated and RNA transposed duplicated genes corresponding to 53 duplicated gene pairs, were also found in the Gossypium hirsutum F-box gene family, being distributed across 22, 13, and 16 chromosomes at low densities (Additional file 1: Figure S1). We note that the number of WGD genes is larger than that of TD, PD, and TRD genes, this finding being consistent with previous studies on the priority of modes of gene duplication in other gene families from Gossypium hirsutum $[40,49,50]$. The results also indicate that the F-box genes of Gossypium hirsutum (AD-genome) mainly originated from interspecific hybridization species Gossypium arboreum (A-genome) and the species Gossypium raimondii (D-genome).

In previous studies, major efforts were spent on identification of the contributions of WGD or TD duplications to the expansion of gene families in Gossypium hirsutum. In contrast, less attention was paid to the potential contributions of other modes of gene duplication such as transposed or dispersed gene duplications. As some recent studies have suggested potential roles of transposed and dispersed gene duplication to plant genome evolution [14], in the present study, we explored all possible duplication modes of the cotton F-box genes, in order to determine their potential contributions to F-box gene family expansion. We found that the order of priority of F-box

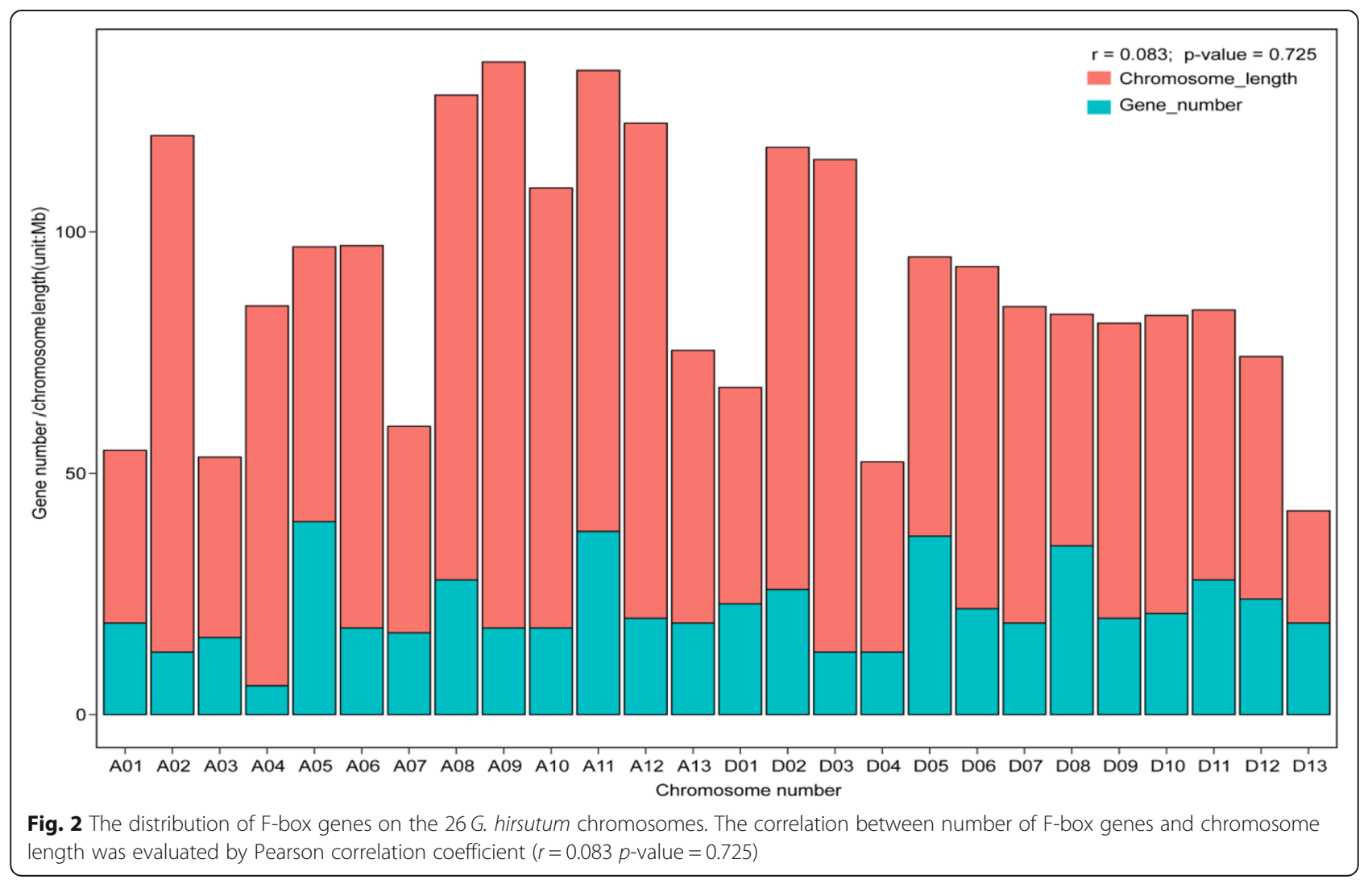




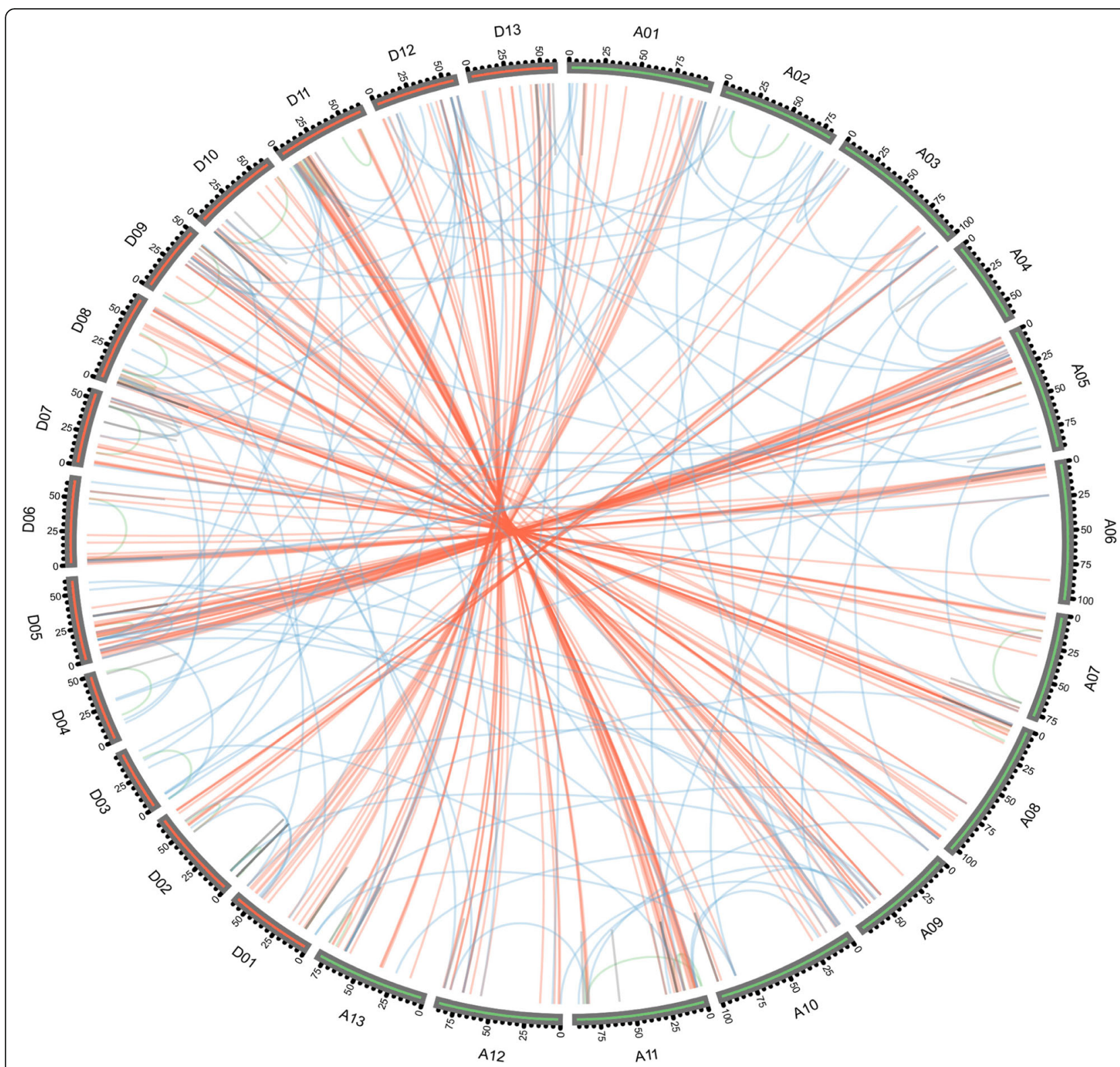

Fig. 3 The synteny pairs of cotton F-box genes from different duplication mode diagrams. The syntenic pairs from whole genome duplication (WGD) were linked by red lines. The brown, green and blue lines represent tandem, proximal and transposed duplication F-box gene-pairs respectively

gene duplication mode is WGD duplication $>$ tandem duplication $>$ transposed duplication >proximal duplication. This is inconsistent with previous studies in other plant species, where the duplication mode priority was found to be WGD duplication > tandem duplication > proximal duplication $>$ transposed duplication [51-53]. Therefore, in addition to whole-genome and tandem gene duplications, other modes of gene duplication, especially transposed duplication, also contribute significantly to the evolutionary expansion of cotton F-box genes. The results from current study therefore provide further insights for understanding the mechanism of expansion of large plant gene families.

To further explore the dynamics of evolution of Gossypium hirsutum F-box genes, comparative studies of the different modes of gene duplication were carried out. This involved estimation of the $\mathrm{Ka}$ (non-synonymous substitutions per site), Ks (synonymous substitutions per site) and $K a / K s$ ratios for each duplication pair, resulting in a measure of the divergence of cotton F-box gene family members. Without excluding extraordinarily 
abnormal values, we found the mean $K a / K s$ ratio for WGD, TD, PD, and TRD were 1.2152, 1.2155, 1.302 and 1.4428 , respectively (Fig. $4 \mathrm{a}-\mathrm{C}$ ), the mean $\mathrm{Ka}$ values for the WGD, TD, PD and TRD were 2.3404, 2.5970, 2.7078 and 1.0963 , respectively, and the mean $K s$ values were 2.3404, 2.3086, 2.3126 and 1.1587, respectively (Fig. 4d-f). We calculated that the average timing of the divergence of WGD, TD, PD, and TRD mapped back to 4.5, 4.4, 4.4, and 2.2 million years ago (MYA), respectively. These results indicate that the surviving WGD, PD, and TD events had undergone a slower sequential or functional divergence for a long period. We further classified the duplicated gene pairs into three groups based on their different selection pressures (Additional file 2: Figure S2). Most of genes from different duplication modes experienced purifying selection $(\mathrm{Ka} / \mathrm{Ks}>1)$, which further demonstrates that cotton F-box genes have undergone positive selection during the polyploidization process.

\section{Gene ontology and organ expressions pattern of F-box protein encoding genes from Gossypium hirsutum}

To further predict the biological functions of the Fbox protein encoding genes in Gossypium hirsutum, Gene Ontology (GO) analysis was performed to probe orthologous genes function based on previous published F-box protein studies. Most of the cotton F-box proteins were identified as involved in the molecular function (GO:0005515) of protein binding, consistent with the established roles that F-box proteins play in post-translational modifications. In addition, 15 of the F-box genes are involved in specific biological processes (BP) (Table 1). We analyzed 15 cotton F-box protein functions further based on the known functions of
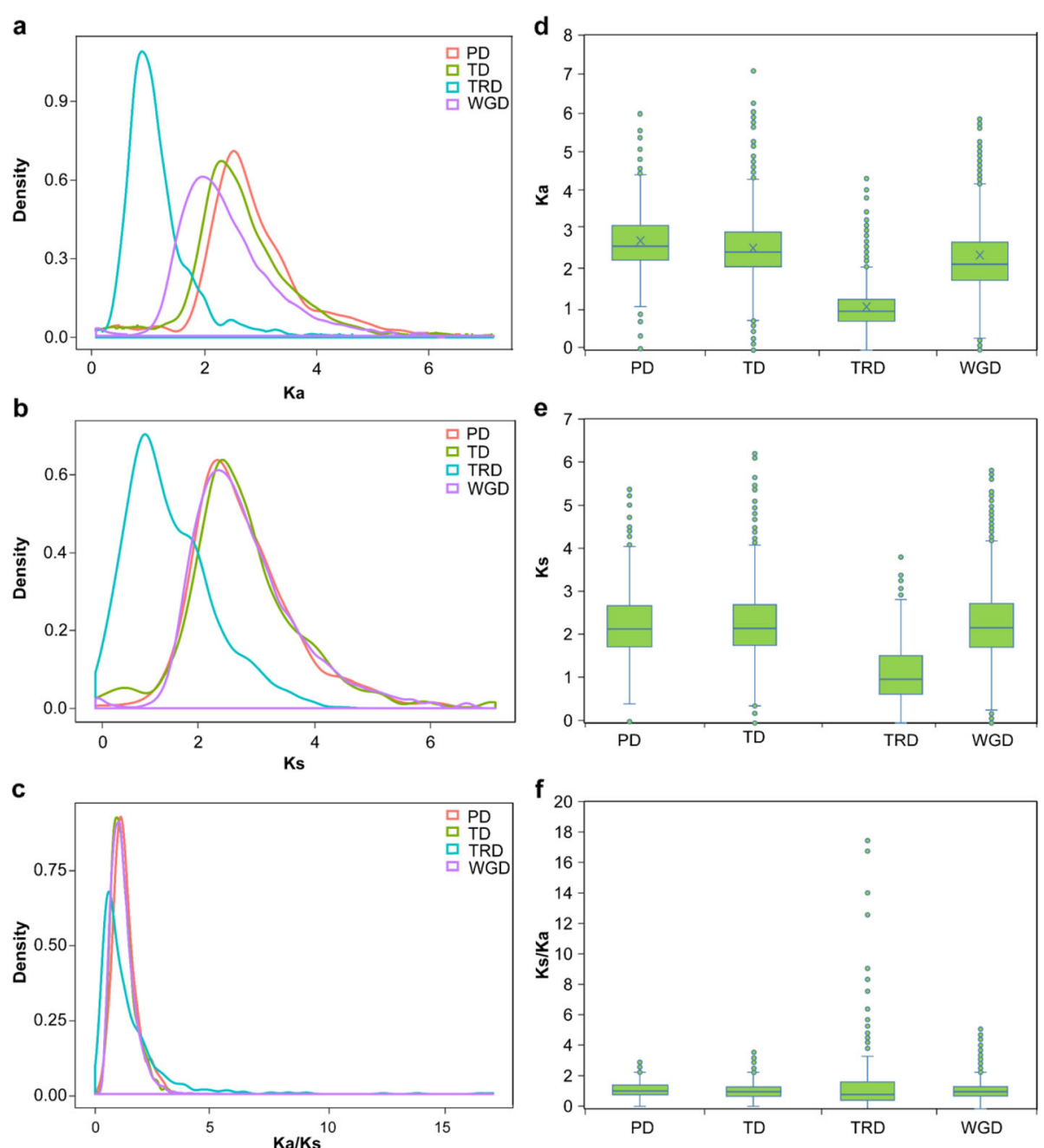

Fig. 4 Evolutionary patterns of gene pairs duplicated by different modes in G. hirsutum. a, d: Ka distributions density and Box plot; $\mathbf{b}$, e: Ks distributions density and Box plot; c, f: Ka/Ks distribution density and Box plot. WGD: whole-genome duplications; TD: tandem duplications; PD: proximal duplications; TRD: transposed duplications; TRD: DNA-transposed duplications 
Table 115 F-box genes involved in biological process (BP) based on the known functions of orthologous Arabidopsis genes

\begin{tabular}{lllll}
\hline Gene duplication group & Principle Transcript ID & GO Number & Arabidopsis ortholog genes & Putative Function of Arabidopsis orthologs \\
\hline WGD & Gh_A08G1127 & 6 & AT3G61590 & protein ubiquitination \\
WGD & Gh_A11G0844 & 6 & AT3G61590 & protein ubiquitination \\
WGD & Gh_D08G1412 & 6 & AT3G61590 & protein ubiquitination \\
WGD & Gh_D11G0986 & 6 & AT3G61590 & protein ubiquitination \\
WGD & Gh_A05G1828 & 5 & AT3G12350 & Biological process \\
WGD & Gh_A13G1079 & 4 & AT5G56180 & actin filament-based process \\
WGD & Gh_D02G1375 & 4 & AT3G60350 & lateral root development \\
WGD & Gh_D07G1180 & 4 & AT5G26960 \\
WGD & Gh_D13G1344 & 4 & AT5G56180 & NA \\
WGD & Gh_A08G1869 & 2 & AT1G55000 & actin filament-based process \\
WGD & Gh_D08G2231 & 2 & AT1G55000 & cell wall macromolecule catabolic process \\
PD & Gh_D02G1602 & 4 & AT3G60350 & cell wall macromolecule catabolic process \\
TRD & Gh_D01G1559 & 4 & AT3G60350 & lateral root development \\
TRD & Gh_D01G1375 & 3 & AT1G68050 & lateral root development \\
TRD & Gh_A10G2188 & 8 & AT3G60160 & positive regulation of flower development \\
\hline
\end{tabular}

orthologous Arabidopsis proteins [12, 14, 54]. Four genes are likely to be involved in protein ubiquitination (Gh_A08G1127, Gh_A11G0844, Gh_D08G1412, Gh_ D11G0986), two genes (Gh_D08G2231, Gh_A08G1869) are involved in cell wall macromolecule catabolic processes, three genes (Gh_D02G1602, Gh_D02G1375, Gh_D01G1559) are involved in lateral root development, one gene (Gh_A10G2188) is involved in the process of response to nematode infections, one gene (Gh_D01G1375) is involved in flower development and two genes were assigned to no known function. In the future, it will therefore be interesting to expand the present study by exploration of their potential regulation roles in development, reproduction, and response to internal or external stimulus of Gossypium hirsutum.

The tissue-specific expression profiles of the 592 cotton F-box protein encoding genes are publicly accessible from a collection of Gossypium hirsutum gene expression databases. In our study, we focused on the expression profiles of cotton F-box genes in the following tissues: Leaf, Root, Stem, Torus, and fibers of different development stages $(5,10,15,20$ dap). F-box genes having expression levels with FPKM values greater than 1 were defined as expressed genes. A further 440 genes with FPKM values greater than 2 and being expressed in at least one of the selected tissues (Additional file 5: Table S5) were defined as high expression genes. This group includes 109 genes from the torus, 91 genes from root, 106 genes from stem and leaf, 60 genes from 5 and 10 dap fibers, and 74 genes from 15 and 20 dap fibers (Additional file 3: Figure S3, Additional file 5: Table S6). $\mathrm{K}$-means analysis resulted in classification of the high expression genes into 5 clusters (high expression in torus, high expression in root, high expression in stem and leaf, high expression in 5 and 10 dap fibers and high expression in 15 and 20 dap fibers) (Fig. 5 and Additional file 4: Figure S4). Among these clusters, 27 genes were found to have high expression in leaf and stem, followed by 22 in root, 20 in torus, 8 in 5 and 10 dap fibers, and 9 in 15 and 20 dap fibers. In each cluster, we noticed that several F-box genes exhibited differential expression in one or more of the cotton plant tissues. These results imply that F-box genes with high expression levels in specific tissues most likely participate in the biological processes specific to that tissue type, whereas the ubiquitously expressed F-box genes may be involved in fundamental cellular processes.

\section{Identification of possible F-box genes in the SCF complexes involved in plant hormone signal transduction pathways}

Most of the F-box proteins can form SCF complexes by binding with the Skp1 protein, and Skp1 further interacts with the scaffold proteins Cullin 1 and Rbx1 to form the major components of classic SCF E3 complexes. Among all the components, F-box proteins serve as the protein recruiting components of the SCF type E3 ubiquitin ligase to determine the specificities of the substrate proteins for ubiquitination and degradation [55]. Studies performed on the model plant Arabidopsis reveal that the common strategy used by plants to precisely respond to hormone signals is by the modulation of the stabilities of key transcription factors by an F-box protein containing ubiquitin ligase [41]. We performed blast analysis against a Gossypium hirsutum cDNA library using Arabidopsis F-box genes as bait that have been 


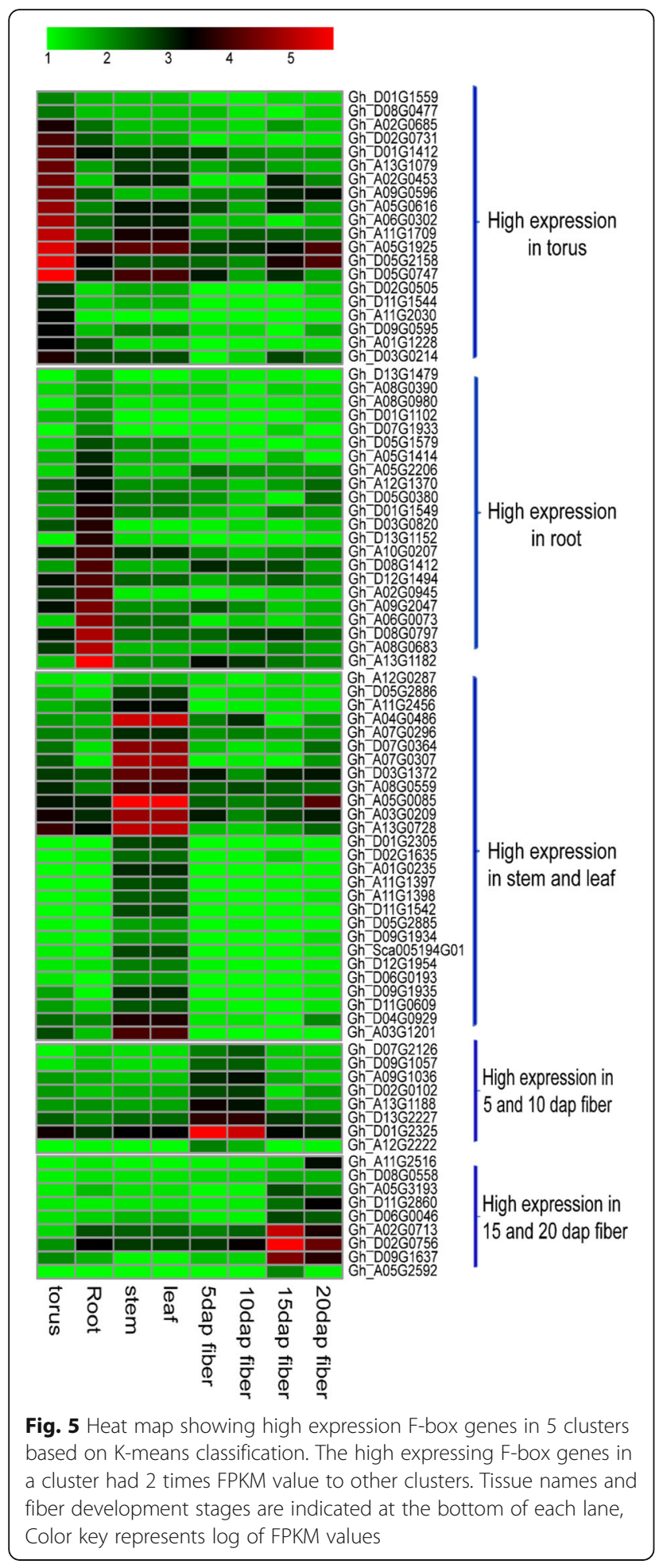

shown to participate in the signal transduction in different hormonal pathways. 43 F-box protein encoding genes were identified as SCF complexes likely involved in different Gossypium hirsutum hormone signal transduction processes (Fig. 6). Among these, 15 cotton Fbox proteins showed greater than $50 \%$ identity to
AtTIR1 protein, the auxin receptor [56], hence likely to contribute to forming the SCF complexes that mediate the AUX/ IAA signaling process, Similarly, 4 proteins showed more than $50 \%$ identity with AtSLP1 protein, the key component of Arabidopsis GA signaling transduction [57], and therefore should form the SCF complexes which likely target DELLA protein for degradation by UPP to mediate gibberellin signaling. Fourteen proteins showed more than $50 \%$ identity with AtEBF2 protein, the important ethylene signaling regulator [58], and so are likely involved in SCF complexes targeting the EIN3 protein to mediate the ethylene signaling. Five COI1 homologous proteins showed more than $50 \%$ identity with AtCOI1 protein, a key regulator of jasmonate signaling [59], and therefore are likely forming the SCF complexes that mediate jasmonate signaling process by UPP. We also identified 5 proteins with more than $50 \%$ identity to the AtMAX2 protein, which plays dual roles in karrikin and strigolactone signaling [60] (Additional file 5: Table S7). Among the AtMAX2 orthologous genes, Gh_A12G2577 and Gh_D12G0880 have been reported to control shoot lateral branching in cotton, consistent with their reported function in Arabidopsis [34]. To confirm that the expression of these potential cotton hormone responsive F-box genes is under influence of hormone homeostatic changes, quantitative real-time PCR analysis was performed. By the end of the course of IAA and GA treatments, representative AtTIR1 homologous genes (Gh_A08G0662, Gh D08G0477 and Gh_D11G1228), and AtSLP1 homologous genes (Gh_A05G2244 and Gh_D05G2503) were all upregulated. In contrast, Gh_A06G0192 was slightly suppressed by $12 \mathrm{~h}$ of GA treatment (Fig. 7). In addition, representative AtMAX2 homologous genes (Gh_D10G0347, Gh A10G0341 and Gh_A06G1896) were all suppressed after $12 \mathrm{~h}$ of ABA treatment, which is consistent with previous report that AtMAX2 expression is suppressed by ABA treatment [42].

\section{Discussion}

F-box family proteins are one of the super protein families in plants, and studies on a limited number of plant F-box proteins have demonstrated that they play diverse roles in various key plant development and physiological processes, including germination [18], floral meristem identity and organ development [20, 61, 62], photomorphogenesis, the circadian clock, flowering time [21, 63-66], regulation of hormone signaling transduction $[41,56-60,67,68]$, plant response to stress conditions $[10,22,23,42,48,69-76]$, plant nutrition usage [77], plant reproductive processes $[16,17]$, and plant primary and secondary metabolism [25-27, 78, 79]. Compared with the vast number of plant F-box genes that have been identified, functional characterizations of the majority of F-box proteins still lags far behind. 


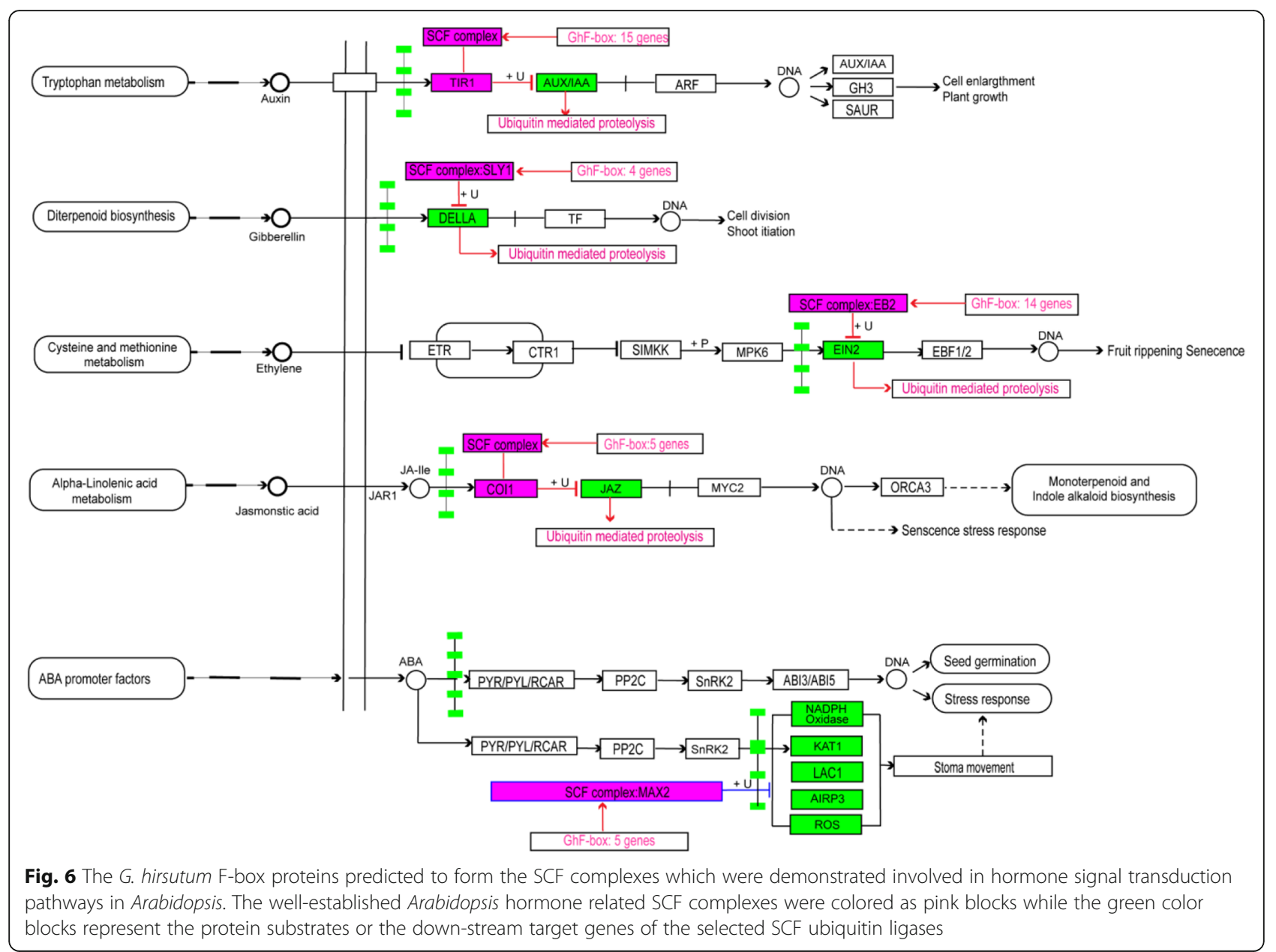

Comprehensive genome-wide identification of plant Fbox family genes is essential before the systematic characterization of their biological functions can be attempted. So far, identification of F-box protein encoding genes at the whole genome level has been reported for a number of plant species, including: Arabidopsis [80, 81], rice [48], grapevine [82], maize [11], apple [13], chickpea [12], Medicago [10], pear [14] and soybean [9]. However, information available for cotton F-box genes and for their roles in the cotton plant life cycle is limited. Here, we first conducted a genome-wide survey of the cotton F-box gene family. Subsequently, their phylogenetic relationships, gene structures, conserved motifs, chromosomal locations, duplication events and their tissue specific expression analysis via in silico analysis of publicly available RNA-sequencing (RNA-seq) database and quantitative reverse-transcription polymerase chain reaction (qRT-PCR) were employed to verify our bioinformatic predictions.

Using the well-established 1808 F-box protein sequences from Arabidopsis, Populus and rice as query sequences [8], 592 F-box protein encoding genes were identified in the new version of the Gossypium hirsutum genome database (http://mascotton.njau.edu.cn). When compared to other sequenced plant species, the Gossypium hirsutum F-box gene family is the third largest, behind rice as the largest (779 family members), and Arabidopsis (692 members) [8]. It is well established that Gossypium hirsutum genome is a result of hybridization of its two parental species Gossypium raimondii and Gossypium arboretum), and upland cotton is a classic model for plant polyploid domestication and genome scale duplication studies. Evidence also suggested that cotton polyploidization process has undergone a subtle gene loss. Previous studies have showed that WGD (Whole Genome Duplication) is the major driving force for the expansion of gene family members in Gossypium hirsutum. In addition to the analysis of contribution of WGD for cotton F-box gene expansion, we also analyzed other modes of gene duplication such as transposed or dispersed gene duplications for their potential contributions to the expansion of the cotton F-box gene family. As compared to the 592 F-box genes found in the Gossypium hirsutum genome, the $300 \mathrm{~F}$-box genes we found 

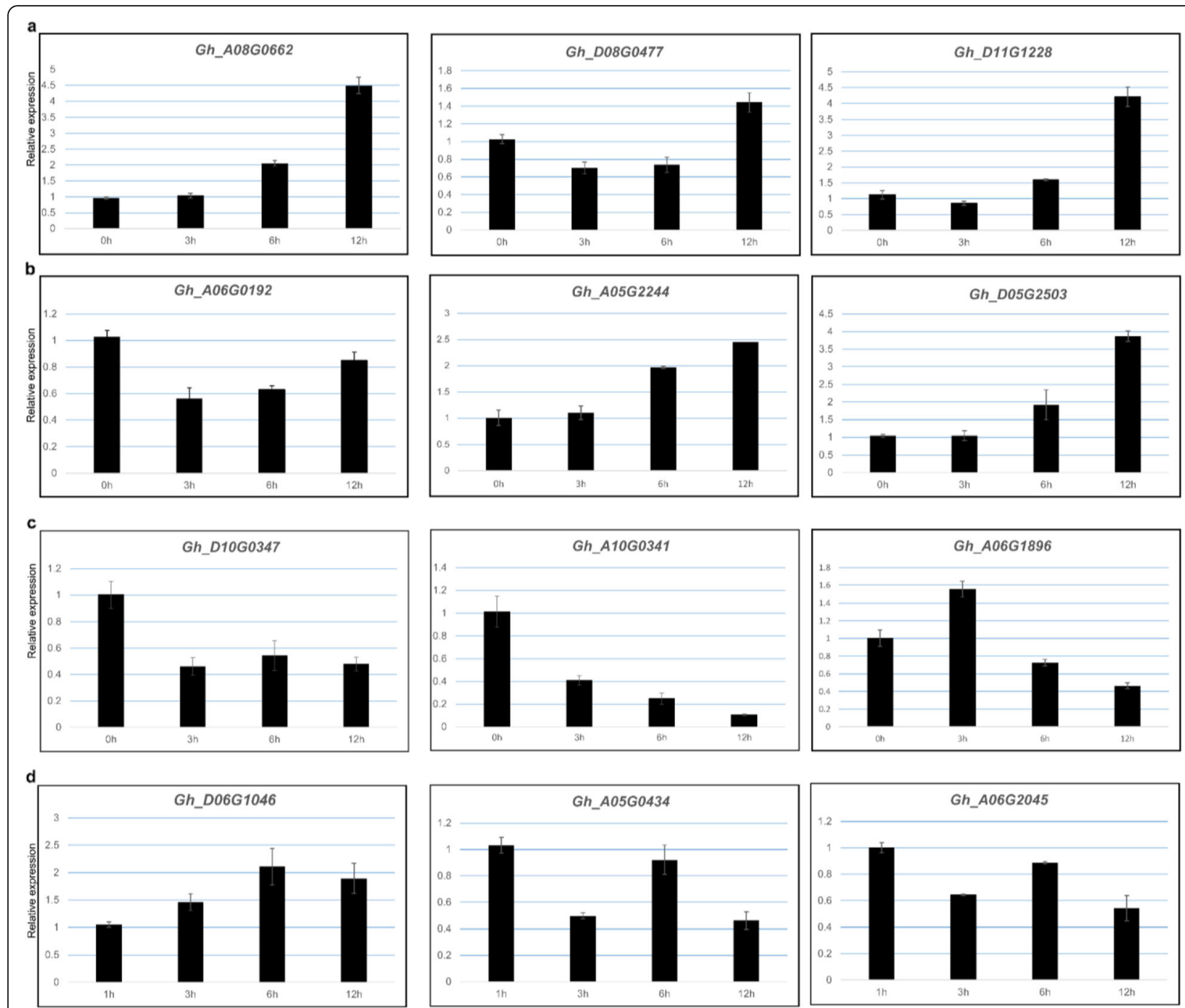

Fig. 7 Expression dynamics of the candidate F-box genes under IBA, GA3, ABA, ACC treatments. Error bars represent SD of three independent biological experiment repeats. The value on the $Y$-axis indicate the relative gene expression levels. The $x$-axis represents the time points when the G. hirsutum leaf samples were collected. a: TIR1 homologous genes expression changes during the course of IBA treatment; b: SLY1 homologous genes expression during the course of GA3 treatment; $\mathbf{c}$ : MAX2 homologous genes expression during the course of $A B A$ treatment; $\mathbf{d}$ : $E B 2$ homologous genes expression during the course of ACC treatment

in the Gossypium raimondii genome and the 282 F-box genes in Gossypium arboreum suggest that, as for other cotton gene families, the F-box gene family also experienced only subtle changes in number in term of family members after the evolution from diploid to tetraploid, and that this gene number change (a slight increase in total F-box gene number which may be the result of contributions by duplication modes other than WGD) indicates the F-box gene family members are indispensable for the enhanced traits of upland cotton and its evolutionary adaptation to variable photic environments.

The well characterized plant F-box proteins so far all contain a functional domain at their $\mathrm{C}$-terminus, and the various $\mathrm{C}$-terminus functional domains were found to be diverse in all the plant species studied so far, including cotton in our current study [6]. Domain analysis of the cotton F-box proteins revealed that a large portion (54\%; 320 out of 592) of the predicted proteins did not have any other known functional domain beyond the Fbox motif itself, and this group of F-box proteins was thereafter designated as Fbox. A similar phenomenon was also found in other plant species (Fig. 1). In the cotton genome, the Kelch domain containing F-box proteins (KFB) represent the most abundant F-box protein subfamily after Fbox (61 in total), similar to the situation in populus and maze, with 35 and 37 subfamily members, respectively (Fig. 1). In addition, Arabidopsis KFBs also form a third most abundant F-box subfamily proteins with 
about 100 members (Fig. 1) [83]. Plant KFBs have been demonstrated to be involved in a range of important biological processes including controlling photoperiodic flowering in Arabidopsis [21, 63], regulation of plant organ fusion and growth [20, 62, 84], controlling Arabidopsis clock progression [64], regulations of rice leaf senescence and yield [79], plant secondary metabolism regulation [25-27] and control of Arabidopsis seed germination [18]. There are no previous reports of the characterizations of cotton KFBs, and it will therefore be interesting to explore the biological functions played by cotton KFBs for their potential contributions to important agricultural traits. Consistent with other plant species, the large subfamilies of cotton F-box proteins also include FBA (46 members), FBD/LRR (34 members), LRR-repeats (9 members) and DUF (18 members) (Fig. 1). The FBA domain-containing F-box proteins have been shown to be involved in regulation of S-RNase-mediated selfincompatibility in Arabidopsis [85]. Almost 10\% (46/ 592) of the cotton F-box proteins belong to FBA subfamily and, based on the established model, the functional significance of this F-box protein subfamily is also worthy to be further explored experimentally in the future.

Plant F-box proteins also have been shown to play critical roles during most of the known hormone signal transduction processes, either serving as the hormone receptors, or as key transcription regulators during hormone perception [41]. Functional characterization of most of the F-box genes so far has been limited in the model plants, such as Arabidopsis, and their homologues in other crop species likely play similar functions. The BLASTP algorithm-based search $(\mathrm{E}<1 \mathrm{e}-10$ and Identities $>50 \%)$ of the F-box genes carried out in our study confirmed the presence of cotton homologues of classic Arabidopsis hormone related Fbox proteins suggesting their probable involvement in similar or identical biological pathways. For example, close homologues of AtTIR1 [56] (Gh_A08G0662, Gh_D08G0477 and Gh_D11G1228), AtCOl1 [59] (Gh_ D04G0642, Gh_Sca006609G01 and Gh_A05G2749), AtSLY1 [86] (Gh_A06G0192, Gh_A05G2244 and Gh_D05G2503), AtMAX2 [42] (Gh_D10G0347, Gh_A10G0341 and Gh A06G1896) and AtEB2 (Gh_D06G1046, Gh_A06G2045 and Gh_A05G0434) which are known to be involved in plant growth and development upon the homeostasis changes of auxin, JA, GA, ABA, and ethylene were readily identified in the cotton F-box gene family (Fig. 6). To investigate the potential functions of these homologues in cotton plants responding to major hormone signaling changes, we performed quantitative RT PCR analysis of the expressions of the representative genes after the cotton leaves were treated with IAA, JA, GA, ABA, and ACC. By the end of IAA treatment, all three AtTIR1 homolog genes expression were significantly up-regulated comparing to the control plants (Fig. 7a). This finding is consistent with the previous published role of AtTIR1 protein as the auxin receptor in Arabidopsis [87]. Although, there is scant evidence showing that auxin can affect AtTIR1 gene transcript levels, our data clearly showed that auxin regulation of plant growth and development is through both transcriptional and post-translational modifications. Jasmonate and related signaling compounds are not only playing the important roles during the processes of plant responses to both biotic and abiotic stresses, but also are crucial for plant growth and development. JAZ1 protein is the key transcription repressor of jasmonate-responsive genes and is degraded by the COI1 containing SCF complex upon JA treatment [59]. Five AtCOI1 homologs were detected in our analyses. GA is another important plant hormone which has been demonstrated to modulate Arabidopsis plant growth and development through the regulation of levels of phosphorylated DELLA protein, the repressors of GA responsive genes, achieved by rapid induction of DELLA protein degradation by $\mathrm{SCF}^{\mathrm{SLY} 1}$ complex [57]. Thus, similar to AtTIR1 and AtCOI1, AtSLY1 is the crucial component of Arabidopsis GA signaling transduction module. We analyzed the potential effects of GA treatment on the expression of the 4 cotton homologues of Arabidopsis SLY1. Two (Gh_A05G2244 and Gh_D05G2503) of the three selected genes showed steady increases over the course of GA treatment. However, Gh_A06G0192 displayed suppressed expression at all the sampling points (Fig. 7c). These data suggest that Gh_A06G0192 may confer its function in a opposing fashion as compared to the remaining two F-box proteins. Further protein-protein interaction experiments will be needed to verify whether any of these SLY1 homologues can interact with the cotton DELLA protein, in order to directly determine whether they are serving as the F-box protein component of the cotton SCFS ${ }^{\mathrm{LY} 1}$ complex participating in the cotton GA signaling pathway. The Arabidopsis F-box protein MORE AXILLARY GROWTH2 (MAX2) has previously been characterized for its role in plant branching and MAX2 appears essential for the perception of the newlycharacterized phytohormone strigolactone, a negative regulator of polar auxin transport in Arabidopsis. In addition, MAX2 has also been shown to be involved in karrikin signaling. But the detailed molecular mechanism awaits to be elucidated [60]. A number of studies also have indicated that MAX2 contributes to plant responses to both biotic and abiotic stresses through modulation of the ABA signaling pathway and that MAX2 gene expression can be influenced by ABA treatment [42, 75, 88]. Bu et al showed that MAX2 expression was suppressed to about $50 \%$ after Arabidopsis seedlings were treated with $50 \mu \mathrm{M}$ ABA for $6 \mathrm{~h}$ [42]. After performing a BLAST search, we identified 5 cotton homologues of $M A X 2$, and three of 
these were selected for modulation of gene expression in response to ABA treatment. Consistent with previous reports, the three cotton $M A X 2$ genes are all suppressed by ABA, especially Gh_A10G0341 expression which steadily decreases during the ABA treatment. The protein substrate for AtMAX2 is not known in Arabidopsis, but considering its important role during plant growth and development (branching would be a particularly beneficial trait directly related the yield of cotton and other crop plant species), it will be interesting to further investigate the functions of cotton MAX2 genes in the future.

\section{Conclusions}

Cotton is the most important crop for renewable fiber production. In this study, a systematic genome-wide analysis of F-box gene family was performed for the first time as the complement to a number of recent cotton genome sequencing projects. As a result, $592 \mathrm{~F}$-box genes were identified and subjected for further structural and phylogenetic characterizations. Our analysis led to the identification of a number of conserved F-box subfamilies present in the cotton genome which show close similarity at the amino acid level to other model and crop plant species. Gene duplication event analysis showed that, from diploid cotton (A-genome parent and $\mathrm{D}$-genome parent) to the allotetraploid AD-hybrid, the expansion of the cotton F-box gene family is exclusively achieved by whole genome duplications (WGD) with only subtle contributions from other gene duplication modes. Digital expression profiles of the F-box genes across different tissues were also explored and several Fbox genes were identified with tissue specific expression patterns implying their possible involvement in the growth and development of selected organs. A combination of homology searches, classic hormone treatments and RT-PCR experiments, identified putative F-box genes likely to be involved in cotton hormone signaling transduction pathways. This study serves as a foundation for the selection and characterization of candidate genes to be used for trait improvement in cotton breeding programs.

\section{Supplementary information}

Supplementary information accompanies this paper at https://doi.org/10. 1186/s12864-019-6280-2.

Additional file 1: Figure S1. The expansion modes of F-box genes in upland cotton. a: The number of F-box genes of different duplication modes. The $\mathrm{x}$-axis represents gene numbers, and the $\mathrm{Y}$-axis represents different duplication modes; b: Distribution of duplicated genes on 26 chromosomes of upland cotton. The $x$-axis represents chromosome numbers, and the $Y$-axis represents the gene number of different duplication modes.

Additional file 2: Figure S2. The proportion of duplicated gene pairs under different (evolution) selection forces. Red dots represent purifying selection where the Ka/Ks value is smaller than "1"; Green dots represent neutral selection where the Ka/Ks equals to "1"; Blue dots represent positive selection where the Ka/Ks is bigger than " 1 ". Abbreviations: WGD (whole-genome duplication); TD (tandem duplication); PD (proximal duplication); TRD (DNA-transposed duplication).

Additional file 3: Figure S3. Organ specific expression of F-box genes in the G. hirsutum. Color scale represents log transformed RPKM values. Light green indicates low expression and red color indicates high expression. Heatmap was generated using R program.

Additional file 4: Figure S4. The optimal number of clusters (K) as determined by 'k-means' clustering. The red line indicated the optimal number of clusters is at five.

Additional file 5: Table S1. Detailed information of FBOX members from Upland cotton. Table S2. Detailed information of F-box members from G. raimondii. Table S3. Detailed information of F-box members from G.arboreum. Table S4. Upland cotton F-box gene numbers for different modes of gene duplication. Table S5. Organ-specific expression patterns of F-box genes. Table S6. Optimal numbers of clusters (K) as determined by 'k-means' clustering. Table S7. F-box gene as the SCF complexes involved in hormone signal transduction based on the homologous gene in Arabidopsis. Table S8. real-time PCR primers.

\section{Abbreviations}

BP: biological process; CP: cellular component; GO: Gene Ontology; MF: molecular function; PD: proximal duplications; RPKM: reads per kilobase per million; TD: tandem duplications; TRD: transposed duplications; WGD: whole-genome duplications

\section{Acknowledgements}

Thanks Professor David Galbraith (The University of Arizona) for providing some critical advice.

\section{Authors' contributions}

ZXB and MYC conceived and designed the experiments, ZSL, TZL, GYT and ZYQ performed the experiments and analyzed the data. ZSL, ZXB and MYC wrote the manuscript. All authors have read and approved the final manuscript.

\section{Funding}

This work was supported by grants from National key research and development program of China (Grant No. 2018YFD0100304. 2018YFD0100302); the National Natural Science Foundation of China (31770300), the Program for Innovative Research Team (in Science and Technology) in University of Henan Province (18IRTSTHN023).

\section{Availability of data and materials}

The data sets supporting the results of this article are included within the article and its additional files.

Ethics approval and consent to participate

Not applicable.

Consent for publication

Not applicable.

\section{Competing interests}

The authors declare that they have no competing interests.

\section{Author details}

'State Key Laboratory of Cotton Biology, Institute of Plant Stress Biology, School of Life Sciences, Henan University, Jinming Street, Kaifeng 475004, China. ${ }^{2}$ College of Biology and Food Engineering, Anyang Institute of Technology, Anyang 455000, China. ${ }^{3}$ Faculty of Science and Engineering, School of Biological \& Marine Sciences, University of Plymouth, Devon, UK. 


\section{Received: 19 December 2018 Accepted: 13 November 2019} Published online: 19 December 2019

\section{References}

1. Gorelik M, Manczyk N, Pavlenco A, Kurinov I, Sidhu SS, Sicheri F. A structurebased strategy for engineering selective ubiquitin variant inhibitors of Skp1Cul1-F-box ubiquitin ligases. Structure. 2018;26(9):1226-36 e1223.

2. Smalle J, Vierstra RD. The ubiquitin 265 proteasome proteolytic pathway. Annu Rev Plant Biol. 2004;55:555-90.

3. Amm I, Sommer T, Wolf DH. Protein quality control and elimination of protein waste: the role of the ubiquitin-proteasome system. Biochim Biophys Acta Molec Cell Res. 2014;1843(1):182-96.

4. Guo XK, Zhang Y, Tu Y, Wang YZ, Cheng WJ, Yang YW. Overexpression of an EIN3-binding F-box protein2-like gene caused elongated fruit shape and delayed fruit development and ripening in tomato. Plant Sci. 2018;272:131-41.

5. Bonhomme M, Andre O, Badis Y, Ronfort J, Burgarella C, Chantret N, Prosperi J-M, Briskine R, Mudge J, Debelle F, et al. High-density genomewide association mapping implicates an F-box encoding gene in Medicago truncatula resistance to Aphanomyces euteiches. New Phytol. 2014;201(4): 1328-42.

6. Lechner E, Achard P, Vansiri A, Potuschak T, Genschik P. F-box proteins everywhere. Curr Opin Plant Biol. 2006;9(6):631-8.

7. Zheng N, Schulman BA, Song LZ, Miller JJ, Jeffrey PD, Wang P, Chu C, Koepp DM, Elledge SJ, Pagano M, et al. Structure of the Cul1-Rbx1-Skp1-F box (Skp2) SCF ubiquitin ligase complex. Nature. 2002;416(6882):703-9.

8. Xu GX, Ma H, Nei M, Kong HZ. Evolution of F-box genes in plants: different modes of sequence divergence and their relationships with functional diversification. Proc Natl Acad Sci U S A. 2009;106(3):835-40.

9. Jia Q, Xiao Z-X, Wong F-L, Sun S, Liang K-J, Lam H-M. Genome-wide analyses of the soybean F-box gene family in response to salt stress. Int J Mol Sci. 2017;18(4):E818.

10. Song JB, Wang YX, Li HB, Li BW, Zhou ZS, Gao S, Yang ZM. The F-box family genes as key elements in response to salt, heavy mental, and drought stresses in Medicago truncatula. Funct Integr Genomics. 2015;15(4):495-507.

11. Jia FJ, Wu BJ, Li H, Huang JG, Zheng CC. Genome-wide identification and characterisation of F-box family in maize. Mol Gen Genomics. 2013;288(11): 559-77.

12. Gupta S, Garg V, Kant C, Bhatia S. Genome-wide survey and expression analysis of F-box genes in chickpea. BMC Genomics. 2015;16:67.

13. Cui HR, Zhang ZR, Lv W, Xu JN, Wang XY. Genome-wide characterization and analysis of F-box protein-encoding genes in the Malus domestica genome. Mol Gen Genomics. 2015;290(4):1435-46.

14. Wang GM, Yin H, Qiao X, Tan X, Gu C, Wang BH, Cheng R, Wang YZ, Zhang SL. F-box genes: genome-wide expansion, evolution and their contribution to pollen growth in pear (Pyrus bretschneideri). Plant Sci. 2016;253:164-75.

15. Gagne JM, Downes BP, Shiu SH, Durski AM, Vierstra RD. The F-box subunit of the SCF E3 complex is encoded by a diverse superfamily of genes in Arabidopsis. Proc Natl Acad Sci U S A. 2002;99(17):11519-24.

16. Kim HJ, Oh SA, Brownfield L, Hong SH, Ryu H, Hwang I, Twell D, Nam HG. Control of plant germline proliferation by SCFFBL17 degradation of cell cycle inhibitors. Nature. 2008;455(7216):1134-7.

17. Gusti A, Baumberger N, Nowack M, Pusch S, Eisler H, Potuschak T, De Veylder L, Schnittger A, Genschik P. The Arabidopsis thaliana F-box protein FBL17 is essential for progression through the second mitosis during pollen development. PLoS One. 2009:4(3):e4780.

18. Majee M, Kumar S, Kathare PK, Wu S, Gingerich D, Nayak NR, Salaita L, Dinkins R, Martin K, Goodin M, et al. KELCH F-BOX protein positively influences Arabidopsis seed germination by targeting PHYTOCHROMEINTERACTING FACTOR1. Proc Natl Acad Sci. 2018:115(17):E4120-9.

19. Baute J, Polyn S, De Block J, Blomme J, Van Lijsebettens M, Inzé D. F-box protein FBX92 affects leaf size in Arabidopsis thaliana. Plant Cell Physiol. 2017:58(5):962-75.

20. Gonzalez-Carranza ZH, Rompa U, Peters JL, Bhatt AM, Wagstaff C, Stead AD, Roberts JA. Hawaiian skirt: an F-box gene that regulates organ fusion and growth in Arabidopsis. Plant Physiol. 2007;144(3):1370-82.

21. Song YH, Smith RW, To BJ, Millar AJ, Imaizumi T. FKF1 conveys timing information for CONSTANS stabilization in photoperiodic flowering. Science. 2012:336(6084):1045-9.

22. Zhao Z, Zhang G, Zhou S, Ren $Y$, Wang W. The improvement of salt tolerance in transgenic tobacco by overexpression of wheat F-box gene TaFBA1. Plant Sci. 2017;259:71-85.
23. Zhou S-M, Kong X-Z, Kang H-H, Sun X-D, Wang W. The involvement of wheat F-box protein gene TaFBA1 in the oxidative stress tolerance of plants. PLoS One. 2015;10(4):e0122117.

24. Bu Q, Lv T, Shen H, Phi L, Wang J, Wang Z, Huang Z, Xiao L, Engineer C, $\mathrm{Kim} T H$, et al. Regulation of drought tolerance by the F-box protein MAX2 in Arabidopsis (1 C W OPEN ). Plant Physiol. 2014;164(1):424-39.

25. Zhang X, Gou M, Liu C-J. Arabidopsis Kelch repeat F-box proteins regulate phenylpropanoid biosynthesis via controlling the turnover of phenylalanine ammonia-lyase. Plant Cell. 2013;25(12):4994-5010.

26. Zhang X, Gou M, Guo C, Yang H, Liu C-J. Down-regulation of Kelch domaincontaining F-box protein in Arabidopsis enhances the production of (poly) phenols and tolerance to ultraviolet radiation. Plant Physiol. 2015;167(2): $337-50$.

27. Zhang X, Abrahan C, Colquhoun TA, Liu C-J. A Proteolytic regulator controlling Chalcone synthase stability and flavonoid biosynthesis in Arabidopsis. Plant Cell. 2017;29(5):1157-74.

28. Koops P, Pelser S, Ignatz M, Klose C, Marrocco-Selden K, Kretsch T. EDL3 is an $\mathrm{F}$-box protein involved in the regulation of abscisic acid signalling in Arabidopsis thaliana. J Exp Bot. 2011;62(15):5547-60.

29. Li Y, Zhang L, Li D, Liu Z, Wang J, Li X, Yang Y. The Arabidopsis F-box E3 ligase RIFP1 plays a negative role in abscisic acid signalling by facilitating ABA receptor RCAR3 degradation. Plant Cell Environ. 2016;39(3):571-82.

30. Li F, Fan G, Wang K, Sun F, Yuan Y, Song G, Li Q, Ma Z, Lu C, Zou C, et al. Genome sequence of the cultivated cotton Gossypium arboreum. Nat Genet. 2014;46(6):567-72.

31. Wendel JF, Grover CE. Taxonomy and evolution of the cotton genus, Gossypium; 2015.

32. Li F, Fan G, Lu C, Xiao G, Zou C, Kohel RJ, Ma Z, Shang H, Ma X, Wu J, et al. Genome sequence of cultivated upland cotton (Gossypium hirsutum TM-1) provides insights into genome evolution. Nat Biotechnol. 2015;33(5):524-30.

33. Wendel JF, Schnabel A, Seelanan T. Bidirectional interlocus concerted evolution following allopolyploid speciation in cotton (Gossypium). Proc Natl Acad Sci U S A. 1995;92(1):280-4

34. Zhao LL, Fang JJ, Xing J, Liu WN, Peng P, Long HX, Zhao JF, Zhang WH, Li $X Y$. Identification and functional analysis of two cotton Orthologs of MAX2 which control shoot lateral branching. Plant Mol Biol Report. 2017;35(5): 480-90.

35. Wei J, Fan S, Meizhen S, Chaoyou P, Shuxun YU. Cloning and expression analysis of GhFBO in Gossypium hirsutum L. Cotton Sci. 2011;23(3):212-8.

36. Zhang TZ, Hu Y, Jiang WK, Fang L, Guan XY, Chen JD, Zhang JB, Saski CA, Scheffler BE, Stelly DM, et al. Sequencing of allotetraploid cotton (Gossypium hirsutum L. acc. TM-1) provides a resource for fiber improvement. Nat Biotechnol. 2015;33(5):531-U252.

37. Wang $Y, L i$ J, Paterson AH. MCScanX-transposed: detecting transposed gene duplications based on multiple colinearity scans. Bioinformatics. 2013;29(11): 1458-60.

38. Wang D, Zhang Y, Zhang Z, Zhu J, Yu J. KaKs_Calculator 2.0: a toolkit incorporating gamma-series methods and sliding window strategies. Genomics Proteomics Bioinformatics. 2010;8(1):77-80.

39. Zhang Z, Li J, Zhao X-Q, Wang J, Wong GK-S, Yu J. KaKs_Calculator: calculating $\mathrm{Ka}$ and $\mathrm{Ks}$ through model selection and model averaging. Genomics Proteomics Bioinformatics. 2006;4(4):259-63.

40. Sun HR, Hao PB, Ma Q, Zhang M, Qin Y, Wei HL, Su JJ, Wang HT, Gu LJ, Wang NH, et al. Genome-wide identification and expression analyses of the pectate lyase (PEL) gene family in cotton (Gossypium hirsutum L.). BMC Genomics. 2018;19:661.

41. McSteen P, Zhao Y. Plant hormones and signaling: common themes and new developments. Dev Cell. 2008;14(4):467-73.

42. Bu Q, Lv T, Shen H, Luong P, Wang J, Wang Z, Huang Z, Xiao L, Engineer C, Kim TH. Regulation of drought tolerance by the F-box protein MAX2 in Arabidopsis. Plant Physiol. 2014;164(1):424-39.

43. Liu Z, Ge X, Yang Z, Zhang C, Zhao G, Chen E, Liu J, Zhang X, Li F. Genomewide identification and characterization of SnRK2 gene family in cotton (Gossypium hirsutum L.). BMC Genet. 2017;18:54.

44. Meng YY, Liu F, Pang CY, Fan SL, Song MZ, Wang D, Li WH, Yu SX. Labelfree quantitative proteomics analysis of cotton leaf response to nitric oxide. J Proteome Res. 2011;10(12):5416-32.

45. Paterson AH, Wendel JF, Gundlach H, Guo H, Jenkins J, Jin D, Llewellyn D, Showmaker KC, Shu S, Udall J, et al. Repeated polyploidization of Gossypium genomes and the evolution of spinnable cotton fibres. Nature. 2012;492(7429):423-7. 
46. Waghmare VN, Rong J, Rogers CJ, Bowers JE, Chee PW, Gannaway JR, Katageri I, Paterson AH. Comparative transmission genetics of introgressed chromatin in Gossypium (cotton) polyploids. Am J Bot. 2016;103(4):719-29.

47. Buggs RJ, Chamala S, Wu W, Tate JA, Schnable PS, Soltis DE, Soltis PS, Barbazuk WB. Rapid, repeated, and clustered loss of duplicate genes in allopolyploid plant populations of independent origin. Curr Biol. 2012;22(3):248-52.

48. Jain M, Nijhawan A, Arora R, Agarwal P, Ray S, Sharma P, Kapoor S, Tyagi AK, Khurana JP. F-box proteins in rice. Genome-wide analysis, classification, temporal and spatial gene expression during panicle and seed development, and regulation by light and abiotic stress. Plant Physiol. 2007; 143(4):1467-83.

49. Zhang B, Liu J, Yang ZE, Chen EY, Zhang CJ, Zhang XY, Li FG. Genome-wide analysis of GRAS transcription factor gene family in Gossypium hirsutum $L$. BMC Genomics. 2018;19:348.

50. Li XH, Liu GY, Geng YH, Wu M, Pei WF, Zhai HH, Zang XS, Li XL, Zhang JF, Yu SX, et al. A genome-wide analysis of the small auxin-up RNA (SAUR) gene family in cotton. BMC Genomics. 2017;18:815.

51. Qiao X, Yin H, Li L, Wang R, Wu J, Wu J, Zhang S. Different modes of gene duplication show divergent evolutionary patterns and contribute differently to the expansion of gene families involved in important fruit traits in pear (Pyrus bretschneideri). Front Plant Sci. 2018;9:161.

52. Li TG, Zhang DD, Zhou L, Kong ZQ, Hussaini AS, Wang D, Li JJ, Short DPG, Dhar N, Klosterman SJ, et al. Genome-wide identification and functional analyses of the CRK gene family in cotton reveals GbCRK18 confers Verticillium wilt resistance in Gossypium barbadense. Front Plant Sci. 2018;9:1266.

53. Wang YP, Tan X, Paterson AH. Different patterns of gene structure divergence following gene duplication in Arabidopsis. BMC Genomics. 2013;14:9.

54. Yang X, Kalluri UC, Jawdy S, Gunter LE, Yin T, Tschaplinski TJ, Weston DJ, Ranjan P, Tuskan GA. The F-box gene family is expanded in herbaceous annual plants relative to Woody perennial plants. Plant Physiol. 2008;148(3): 1189-200.

55. Petroski MD, Deshaies RJ. Function and regulation of cullin-RING ubiquitin ligases. Nat Rev Mol Cell Biol. 2005;6(1):9-20.

56. Kepinski S, Leyser $\mathrm{O}$. The Arabidopsis F-box protein TIR1 is an auxin receptor. Nature. 2005;435(7041):446-51.

57. Dill A, Thomas SG, Hu J, Steber CM, Sun TP. The Arabidopsis F-box protein SLEEPY1 targets gibberellin signaling repressors for gibberellin-induced degradation. Plant Cell. 2004;16(6):1392-405.

58. Binder BM, Walker JM, Gagne JM, Emborg TJ, Hemmann G, Bleecker AB, Vierstra RD. The Arabidopsis EIN3 binding F-box proteins EBF1 and EBF2 have distinct but overlapping roles in ethylene signaling. Plant Cell. 2007; 19(2):509-23.

59. Thines B, Katsir L, Melotto M, Niu Y, Mandaokar A, Liu G, Nomura K, He SY, Howe GA, Browse J. JAZ repressor proteins are targets of the SCFCOI1 complex during jasmonate signalling. Nature. 2007:448(7154):661-5.

60. Nelson DC, Smith SM. F-box protein MAX2 has dual roles in karrikin and strigolactone signaling in Arabidopsis thaliana. Plant Signal Behav. 2011; 108(9):8897-902.

61. Levin JZ, Meyerowitz EM. UFO: an Arabidopsis gene involved in both floral meristem and floral organ development. Plant Cell. 1995;7(5):529-48.

62. Zhang X, Jayaweera D, Peters J, Szecsi J, Bendahmane M, Roberts J, González-Carranza Z. The Arabidopsis thaliana F-box gene HAWAlIAN SKIRT is a new player in the microRNA pathway. PLoS One. 2017;12(12):e0189788.

63. Imaizumi T, Schultz TF, Harmon FG, Ho LA, Kay SA. FKF1 F-box protein mediates cyclic degradation of a repressor of CONSTANS in Arabidopsis. Science. 2005;309(5732):293-7.

64. Baudry A, Ito S, Song YH, Strait AA, Kiba T, Lu S, Henriques R, Pruneda-Paz $J$, Chua NH, Tobin EM, et al. F-box proteins FKF1 and LKP2 act in concert with ZEITLUPE to control Arabidopsis clock progression. Plant Cell. 2010; 22(3):606-22.

65. Sawa M, Nusinow D, Kay S, Imaizumi T. FKF1 and GIGANTEA complex formation is required for day-length measurement in Arabidopsis. Science. 2007:318(5848):261-5.

66. Chae E, Tan QK, Hill TA, Irish VF. An Arabidopsis F-box protein acts as a transcriptional co-factor to regulate floral development. Development. 2008; 135(7):1235-45.

67. Sasaki A, Itoh H, Gomi K, Ueguchi-Tanaka M, Ishiyama K, Kobayashi M, Jeong D-H, An G, Kitano H, Ashikari M. Accumulation of phosphorylated repressor for gibberellin signaling in an F-box mutant. Science. 2003; 299(5614):1896-8.
68. Mouchel CF, Leyser O. Novel phytohormones involved in long-range signaling. Curr Opin Plant Biol. 2007;10(5):473-6.

69. Kim HS, Delaney TP. Arabidopsis SON1 is an F-box protein that regulates a novel induced defense response independent of both salicylic acid and systemic acquired resistance. Plant Cell. 2002;14(7):1469-82.

70. Calderon-Villalobos LI, Nill C, Marrocco K, Kretsch T, Schwechheimer C. The evolutionarily conserved Arabidopsis thaliana F-box protein AtFBP7 is required for efficient translation during temperature stress. Gene. 2007; 392(1-2):106-16.

71. Cao Y, Yang Y, Zhang H, Li D, Zheng Z, Song F. Overexpression of a rice defense-related F-box protein gene OsDRF1 in tobacco improves disease resistance through potentiation of defense gene expression. Physiol Plant. 2008;134(3):440-52.

72. Zhang YE, Xu W, Li Z, Deng XW, Wu W, Xue Y. F-box protein DOR functions as a novel inhibitory factor for Abscisic acid-induced Stomatal closure under drought stress in Arabidopsis. Plant Physiol. 2008;148(4):2121-33.

73. Bao Y, Song W-M, Jin Y-L, Jiang C-M, Yang Y, Li B, Huang W-J, Liu H, Zhang $\mathrm{H}-\mathrm{X}$. Characterization of Arabidopsis tubby-like proteins and redundant function of AtTLP3 and AtTLP9 in plant response to ABA and osmotic stress. Plant Mol Biol. 2014;86(4-5):471-83.

74. Zhou S, Sun $X$, Yin S, Kong $X$, Zhou S, Xu Y, Luo Y, Wang W. The role of the F-box gene TaFBA1 from wheat (Triticum aestivum L.) in drought tolerance. Plant Physiol Biochem. 2014;84:213-23.

75. Piisilä M, Keceli M, Brader G, Jakobson L, Jõesaar I, Sipari N, Kollist H, Palva E, Kariola T. The F-box protein MAX2 contributes to resistance to bacterial phytopathogens in Arabidopsis thaliana. BMC Plant Biol. 2015;15:53.

76. Stefanowicz K, Lannoo N, Zhao Y, Eggermont L, Van Hove J, Al Atalah B, Van Damme E. Glycan-binding F-box protein from Arabidopsis thaliana protects plants from Pseudomonas syringae infection. BMC Plant Biol. 2016; 16(1):213.

77. Chen ZH, Jenkins Gl, Nimmo HG. Identification of an F-box protein that negatively regulates $\mathrm{P}(\mathrm{i})$ starvation responses. Plant Cell Physiol. 2008;49(12): 1902-6.

78. Kim YY, Jung KW, Jeung JU, Shin JS. A novel F-box protein represses endothecial secondary wall thickening for anther dehiscence in Arabidopsis thaliana. J Plant Physiol. 2012;169(2):212-6.

79. Chen Y, Xu Y, Luo W, Li W, Chen N, Zhang D, Chong K. The F-box protein OsFBK12 targets OsSAMS1 for degradation and affects pleiotropic phenotypes, including leaf senescence, in Rice. Plant Physiol. 2013;163(4): 1673-85.

80. de Ruijter NC, Malhó R. F-box proteins in Arabidopsis. 2000.

81. Kuroda H, Takahashi N, Shimada H, Seki M, Shinozaki K, Matsui M. Classification and expression analysis of Arabidopsis F-box-containing protein genes. Plant Cell Physiol. 2002;43(10):1073-85.

82. Paquis S, Mazeyrat-Gourbeyre F, Fernandez O, Crouzet J, Clément C, Baillieul F, Dorey S. Characterization of a F-box gene up-regulated by phytohormones and upon biotic and abiotic stresses in grapevine. Mol Biol Rep. 2011;38(5):3327-37.

83. Sun Y, Zhou X, Ma H. Genome-wide analysis of Kelch repeat-containing Fbox family. J Integr Plant Biol. 2007;49(6):940-52.

84. González-Carranza Z, Zhang X, Peters J, Boltz V, Szecsi J, Bendahmane M, Roberts J. HAWAIIAN SKIRT controls size and floral organ number by modulating CUC1 and CUC2 expression. PLoS One. 2017;12(9):e0185106.

85. Wang L, Dong L, Zhang Y, Zhang Y, Wu W, Deng X, Xue Y. Genome-wide analysis of S-locus F-box-like genes in Arabidopsis thaliana. Plant Mol Biol. 2004;56(6):929-45.

86. Davière J-M, Achard P. Gibberellin signaling in plants. Development. 2013; 140(6):1147-51.

87. Tan X, Calderon-Villalobos L, Sharon M, Zheng C, Robinson C, Estelle M, Zheng N. Mechanism of auxin perception by the TIR1 ubiquitin ligase. Nature. 2007:446(7136):640-5.

88. An JP, Li R, Qu FJ, You CX, Wang XF, Hao YJ. Apple F-box protein MdMAX2 regulates plant photomorphogenesis and stress response. Front Plant Sci. 2016;7(1235):1685.

\section{Publisher's Note}

Springer Nature remains neutral with regard to jurisdictional claims in published maps and institutional affiliations. 